\title{
Understanding the Effect of Hydrogen Surface Passivation and Etching on the Shape of Silicon Nanocrystals
}

\author{
T. Hawa ${ }^{\dagger}$ and M. R. Zachariah* \\ Departments of Mechanical Engineering and Department of Chemistry and Biochemistry, University of \\ Maryland, College Park, and National Institute of Standards and Technology, Gaithersburg, Maryland \\ Received: January 26, 2008; Revised Manuscript Received: June 2, 2008
}

\begin{abstract}
One of the significant challenges in the use of nanocrystals, is the control of crystal shape when grown from the gas-phase. Recently, the Kortshagen group has succeeded in generating cubic Si nanocrystals in a nonequilibrium plasma. In this paper we consider the energetics of various shaped Si nanocrystals, and the role that hydrogen surface termination plays. We consider cube, truncated octahedron, icosahedron, and spherical shapes for both bare and hydrogen coated silicon nanocrystals for sizes between 2 and $10 \mathrm{~nm}$. From our molecular dynamics (MD) simulations, show that for bare Si crystals, icosahedron crystals are the most energetically stable, and cubic the least. On the other hand, when hydrogenated, the cubic structure comes about because 1) the cubic structure is energetically favored when hydrogen terminated and 2) the plasma that operates with hydrogen also provides a steady source of hydrogen atoms for etching.
\end{abstract}

\section{Introduction}

Nanoclusters and nanocrystals are considered one of the fundamental building blocks for the creation of nanostructures and associated devices. In particular for optoelectronic application quantum confinement effects and the role of surface states for crystals smaller than $10 \mathrm{~nm}$ are of particular interest. ${ }^{1-4}$ Fabrication of the desired shape with a desired structure, is seen as one of the major challenge in robust implementation of nanoscience to a nanotechnology.

Some recent studies have focused on crystalline silicon nanoclusters as an attractive material due to their size and shape dependent properties for electronic applications such as single electron transistors, ${ }^{5}$ vertical transistors, ${ }^{6}$ and floating gate memory devices. ${ }^{7-10}$ Silicon nanocrystals are also found application for photonic devices. ${ }^{11-22}$

A variety of methods to prepare silicon nanocrystals has been reported in the literature. Liquid phase processes produce narrow crystal size distributions, and enable surface passivation using organic polymers. ${ }^{13,15,23-28}$ For example, the reduction of silicon tetrachloride with sodium naphthalenide in 1,2-dimethoxyethane at room temperature, followed by termination of with $n$ butyllithium, produced faceted tetrahedral silicon nanocrystallites. ${ }^{25}$ In contrast gas-phase processes, which typically have high processing rates, are considerably more important industrially at the moment,. The high temperature thermal decomposition of silane in furnace flow reactors is one of primary methods for the formation of silicon nanocrystals. ${ }^{11,26}$ The materials produced however, are typically of a wide size distribution, highly aggregated and contain a significant amorphous character. In general it is extremely difficult to fabricate crystals in desired shape in the gas-phase aerosol processes. Recently, however, the Kortshagen group succeeded in developing a plasma synthesis approach which generates nonagglomerated nanocrystals with a highly monodisperse size distribution. ${ }^{27}$ Their approach uses a high plasma density, and a filamentary radio

\footnotetext{
* To whom correspondence should be addressed. E-mail: mrz@ umd.edu.

$\dagger$ Current address: School of Aerospace and Mechanical Engineering, The University of Oklahoma, Norman, Oklahoma.
}

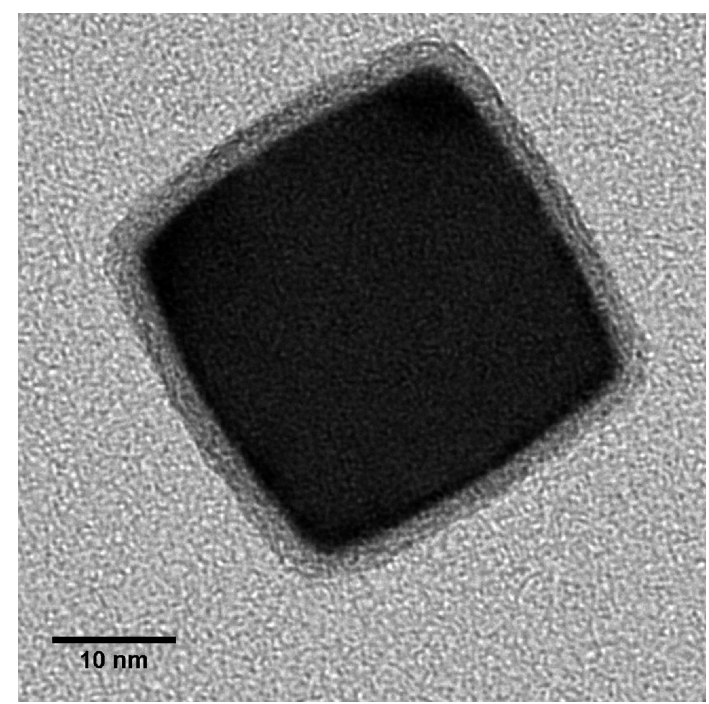

Figure 1. Single crystal, cubic shape silicon nanocrystals produced in a nonthermal, constricted capacitive plasma. ${ }^{54}$

frequency discharge, from which they produced cubic shaped silicon nanocrystals (see Figure 1). This leads naturally to ask what enables the formation of these crystals.

Various numerical methods can be applied to find low energy configurations of nanocrystals. Such examples are amorphization-recrystallization, ${ }^{28}$ simulated annealing, ${ }^{29}$ and genetic algorithms. ${ }^{30}$ For small crystals ab initio methods can be applied, while for larger crystals empirical potential models are often employed. For example, first-principle calculations for bare $\mathrm{Si}$ crystals showed that icosahedral nanocrystals are more stable than truncated crystals. ${ }^{31}$ Kinetic Monte-Carlo simulations have also shown morphological changes. ${ }^{32}$ Barnard and Zapol developed a thermodynamic model to describe the shape of nanocrystals as a function of size..$^{33}$ They have shown that hydrogenated surface has an important impact on nanocrystal morphology and phase stability. MD simulation is an alternative method, describing gold and silver nanocrystal shapes under 
different conditions, such as crystal size $e^{34,35}$ and temperature..$^{36,37}$ However, to our knowledge, the effect of hydrogen termination of Si surface on Si nanocrystals using MD simulations has not been reported.

This paper is focused on understanding how and why the unique crystallites generated by the Kortshagen group come about. We will use MD simulations to track the evolution of crystal morphology, to analyze the stability of various crystal shapes, and to study etch rate of various silicon surfaces. We provide insight into the relationship between the stability analysis and the etch rate of substrates, and propose the mechanism consistent with the generation of cubic silicon nanocrystals.

\section{Computational Model and Simulation Procedure}

This study involves atomistic simulations using classical molecular dynamics. For this work we use the reparametarized KTS interatomic potential for the silicon-hydrogen system developed by Hawa and Zachariah (HZ). ${ }^{38}$ This interatomic potential for silicon was originally developed by Stillinger and Weber $(\mathrm{SW})^{39}$ and extended by Kohen et al. ${ }^{40}$ to include $\mathrm{Si}-\mathrm{H}$ and $\mathrm{H}-\mathrm{H}$ interactions. Similar sets of potential energy functions have also been developed by Murty and Atwater, ${ }^{41}$ Ohira et al. ${ }^{42-44}$ and Ramalingam et al. $^{45}$ where a Tersoff type potential $^{46-49}$ was extended to describe interatomic interactions in the Si:H system. This extended version of the Tersoff potential has been tested successfully for its accuracy in describing the $\mathrm{Si}: \mathrm{H}$ system in several earlier studies, however the simulation of liquid silicon was not well described by the potential. ${ }^{46} \mathrm{By}$ contrast, the extended SW potential (HZ) was designed to describe interactions in both solid and liquid forms of silicon. Since most synthesis processes leading to crystal formation occur at high temperature, crystal growth by coalescence is dominated by liquid-like characteristics, and the accuracy of the SW potential increases with increasing crystal size or temperature, we use this potential for our investigations. The $\mathrm{HZ}$ potential energy is a sum of two and a three-body interactions, and the details of the model and its parameters are given in the literature (Hawa and Zachariah). ${ }^{38}$

All simulations were run on a linux cluster, running up to 16 processors. Atom trajectories were determined by integrating the classical equations of motion using the velocity form of the Verlet algorithm, ${ }^{50}$ with rescaling of atomic velocities at each time step to achieve temperature control. Time steps of 0.5 and 0.05 fs were typically used for pure silicon crystals, and for hydrogen coated crystals, respectively, to ensure energy conservation, and the Verlet neighbor list with parallel architecture was employed in all the simulations, with a neighbor list renewal every 10 steps. The simulations take place in an infinitely large cavity for the stability studies and in a square computation domain of $4.2 \times 3.8 \times 3.2 \mathrm{~nm}$ with periodic boundary conditions in $x$ and $y$ directions for the etching studies.

\section{Results and Discussion}

1. Stability of Nanocrystals. One of the questions we hope to clarify is if the Kortshagen cubic crystals are formed because they are the thermodynamically stable state, or if kinetic conditions govern the observed morphology. We prepared four different shapes (cube, sphere, truncated octahedron (TO), icosahedron (ICOS)) of Si crystal crystals at $300 \mathrm{~K}$ with and without hydrogen coating for all shapes. For hydrogenated crystals, we placed a $\mathrm{H}$ atom on each surface $\mathrm{Si}$ atom. However, for those crystals with (100) surfaces, we placed two H atoms on these surface atoms to create fully coated crystals. In the

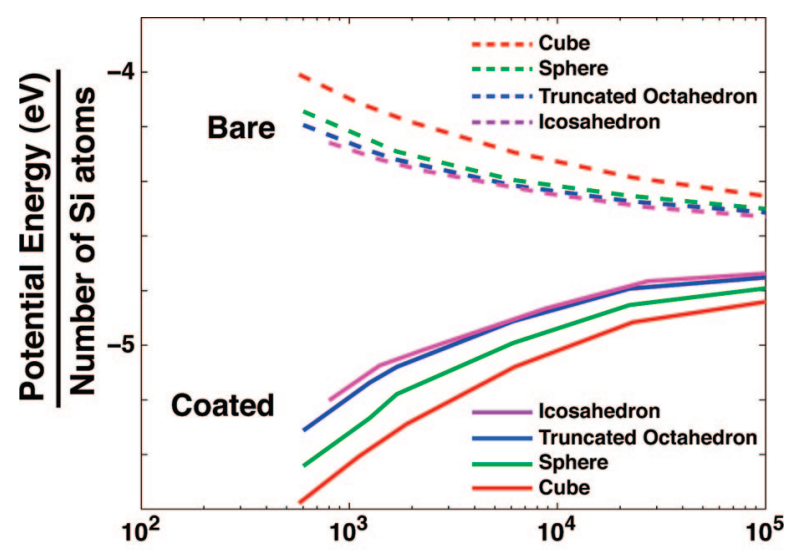

Number of Si atoms in a Cluster

Figure 2. Potential energy for bare and hydrogen terminated spherical, cubic, and truncated octahedron silicon nanocrystals as a function of size at $300 \mathrm{~K}$.

crystal preparation process the simulations were switched from a constant temperature to a constant energy calculation for 20 ps. If the average temperature of the crystal deviated by more than $10 \mathrm{~K}$ over this period, the equilibration process was repeated until the crystal temperature deviated by less than 10 $\mathrm{K}$. The total potential energy of these crystals were averaged by collecting 200 snapshots over 40 ps. Dashed lines in Figure 2 describe the potential energy per $\mathrm{Si}$ atom for all four shapes of Si crystals as a function of crystal size (600-200 000 atoms) at $300 \mathrm{~K}$ temperature. The results indicate that for bare $\mathrm{Si}$ crystals the ICOS is the most stable shape, TO is slightly less stable than ICOS, and the cube is the least stable shape, for all sizes in our study. The largest energy change is observed for the smaller crystals due to the effects of surface to volume ratio, (i.e., large relative number of surface atoms), and the energy asymptotically approaches the bulk values as crystal size increases. In general, crystals tend to minimize their surface area so that they can minimize the number of free bonds on the surface of their crystals. Both (110) surfaces of the ICOS, and the major (111) surfaces of the truncated octahedron crystals have one free bond per surface Si atom. On the other hand, (100) surfaces, gives a cubic shape, and have two free bonds per surface Si atom. On the basis of just surface area, the ICOS and the truncated octahedron have a smaller surface to volume ratio, and should be more stable than the cube. Moreover, each facet of the cube generates twice as many free bonds as that of the ICOS and the truncated octahedron. Thus, the ICOS and the truncated octahedron are the more stable shape than the cube.

We now turn our attention to the dynamics of crystal morphology evolution. According to the previous static stability analysis, the ICOS is the most stable shape and the TO is the second most stable shape in the range of the crystal size studies. We begin by observing the evolution of an initial $4 \times 4 \times 4$ $\mathrm{nm}$ cube containing $2980 \mathrm{Si}$ atoms. The kinetics of crystal transformation is a highly activated process, and can be slow on the time scale typical for an MD simulation. In order to accelerate the transition process, the crystal temperature is increased to $1200 \mathrm{~K}$, which is around the melting point for this size of Si crystal, and then quenched to $800 \mathrm{~K}$ linearly in a 10 ps interval. The crystal is held at $800 \mathrm{~K}$ for another $10 \mathrm{ps}$, and then its temperature is cycled back to $1200 \mathrm{~K}$ again. This annealing/quenching process is repeated until the transition of the crystal morphology is stabilized. The choice of this approach is not arbitrary, but rather qualitatively consistent with what is believed to occur in these nonequilibrium plasmas. Particles 

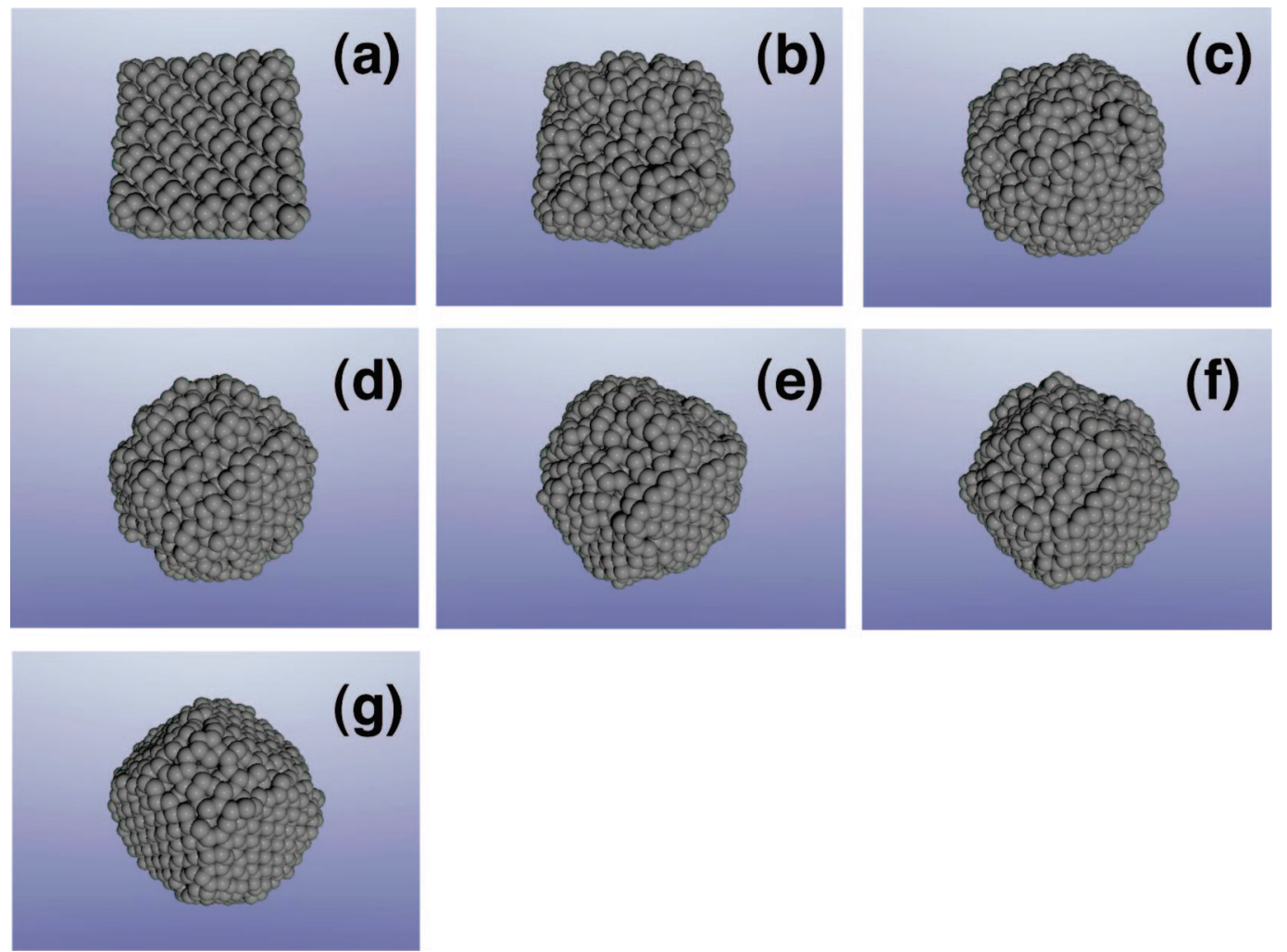

Figure 3. Transition dynamics from a cube to a truncated octahedron.

grown in nonequilibrium plasma are bombarded by very hot electrons $(>20000 \mathrm{~K})$, and relatively cold ions and neutrals ( $<900 \mathrm{~K}$ in the Kortshagen plasma experiment). It is reasonable to expect that the particle temperature will undergo rapid heating and cooling events, and Korshagen shows in Figure 5 of ref 51 particle temperature oscillations between 500 and $900 \mathrm{~K}$. Our MD simulation assumes the same essential mechanism, but with a much faster annealing/quenching cycle, consistent with MD simulation limits. Figure 3 presents the temporal evolution for the cubic Si crystal during this annealing/quenching process. Immediately after the crystal temperature is increased, the surface of the cube begins melting (Figure 3b). The lower-right corner of the cube evolves slowly to form the (111) facet of the TO (Figure 3c). While the (111) facet grows in Figure 3d, another (111) facet emerges next to the first facet (Figure 3e). The crystal evolves into the TO (Figure 3f) and all (111) facets are well developed on the crystal although the (100) surfaces are not fully constructed (Figure 3g). Since (111) facets are known to be more stable than (110) and (100) facets, (111) facets are seen to be generated first. Because the number of Si atoms containing in this crystal is not an exact magic number to construct the TO, the less stable (100) facets cannot be generated fully during the simulation. The results of this simulation also corroborate the higher stability of the TO over the cube as discuss in the stability study. Note that the previous stability study concluded that the ICOS shape is more stable than the TO. However, the ICOS shape is made of 20 tetrahedron building blocks and interfaces of these tetrahedron crystals create plane defects (or grain boundaries). The plane defects grow in a radial direction and the interface area grows exponentially as crystal sizes. Generation of the ICOS shaped Si crystal requires the above-mentioned special symmetric plane defects.

2. Hydrogen Surface Coverage. Both static stability analysis and dynamics simulation suggest that generation of cubic Si crystals is not thermodynamically and possibly not kinetically favored in a vapor phase process. Nevertheless the experimental evidence from the Kortshagen's group is quite clear that they can be produced with high $(65 \%)$ yield. Thus far in our study we have evaluated pure Si structures. However, in the synthesis plasma, hydrogen is present and crystals are known to be hydride terminated. ${ }^{52}$ Furthermore, one should also expect considerable hydrogen dissociation to yield atomic hydrogen, which should be very reactive with the silicon crystals being nucleated. To address the potential influence of hydrogen we begin that hydrogen atoms are terminated on the surface of the Si crystals. We start with the Si crystals used for the static stability analysis, where we have generated cube, sphere, ICOS, and TO shapes and sizes (600-200 000 atoms), and hydrogen terminates the crystals.

Solid lines in Figure 2 describe computed potential energy per $\mathrm{Si}$ atoms for the four shapes of crystals as a function of crystal sizes. The results indicate that for hydrogen terminated Si crystals, the cube is the most stable shape, and that the ICOS is the least stable shape among these four shapes, for all sizes in our study. A large energy change is also observed as a function of size due to the surface to volume ratio, however in contrast to the bare silicon case, the energy increases as the crystal size increases because the energy per atom is calculated only by $\mathrm{Si}$ atoms, not for total $(\mathrm{Si}+\mathrm{H})$ atoms. As we observed in bare $\mathrm{Si}$ crystals, the energy asymptotically approaches to the bulk values as crystal size increases. In general, crystals tend to minimize the number of free bonds on the surface; however, there are no free bonds on the surface of the hydrogen terminated surface. In this case, since the (110) surfaces of the ICOS crystals have one free bond per surface $\mathrm{Si}$ atoms, only one hydrogen atom can be terminated per Si surface atom. On the other hand, the (100) surfaces have two free bonds per surface $\mathrm{Si}$ atoms, and two hydrogen atoms can be terminated per $\mathrm{Si}$ surface atom. 


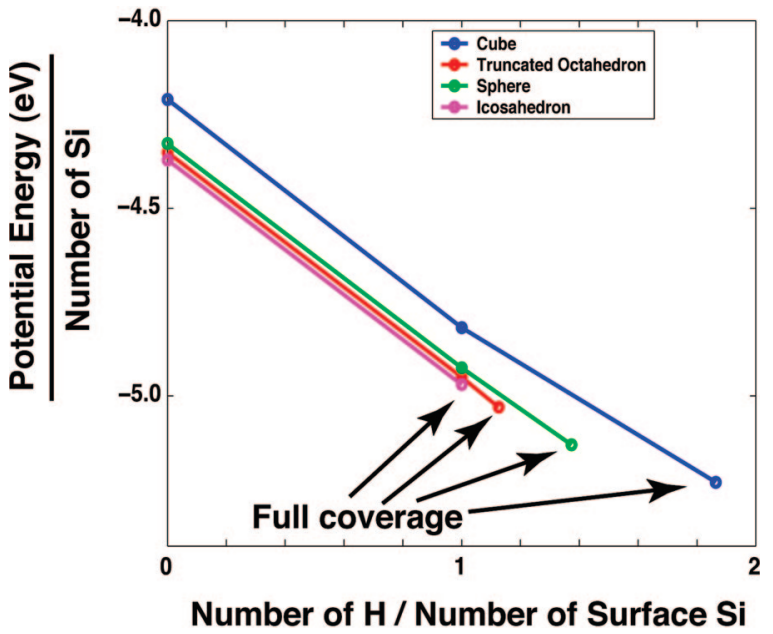

Figure 4. Effect of hydrogen concentration on the surface of nanocrystals.

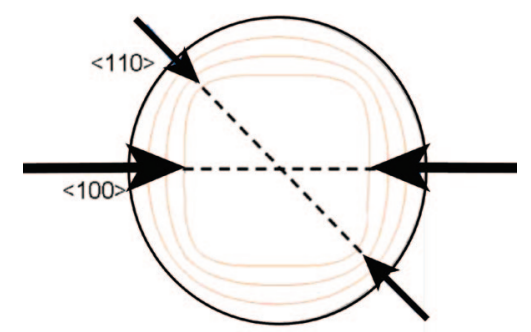

Figure 5. Generation of a cube by hydrogen etch of an originally spherical crystal. The slower etch rate in (110) and (111) directions generate the emergence of (100) surface on the crystal.

Figure 4 shows the surface coverage dependence on the potential energy for about $2500 \mathrm{Si}$ atoms cube, sphere, ICOS, and TO crystals. The plot clearly shows that when hydrogen atom termination per $\mathrm{Si}$ surface atom $<1$, the order in crystal stability is the same as for bare crystals, i.e., the ICOS is the most stable and the cube is the least stable shape. The crystal surface becomes fully $\mathrm{H}$ covered at $\mathrm{H} / \mathrm{Si}$ surface at 1.0, and its energy per atom is $-4.95 \mathrm{eV}$. The cube becomes fully $\mathrm{H}$ coated at $\mathrm{H} / \mathrm{Si}$ surface at 1.9 and its energy per atom is $-5.2 \mathrm{eV}$. Thus, we conclude that this plasma process provided a fast kinetic pathway to surface passivate silicon, and it is this hydrogen surface coverage that promotes the formation of the cube structure. One important point is that the plasma is highly nonequilibrium in that while the thermal temperatures is moderately low, the electron temperatures are several eV's in energy, and this allows for the formation of a high concentrations of dissociated hydrogen at thermal temperatures well below $1000 \mathrm{~K}$.

3. Etching: A Method to Go from Spheres to Cubes. The static stability analysis showed that the hydrogen coated cube is the most stable shape. However, vapor phase nucleation is a highly nonlinear process which usually leads at least initially to amorphous materials, usually spherical in shape. How then does the spherical shape morph into the cube? It is known that dissociated hydrogen will etch silicon. About a decade ago, Sasaki and Takada, prepared various single crystal surfaces of Si (100, 110, and 111), and dosed then with hydrogen atoms to study etch rates. ${ }^{53}$ They found that the etch rate followed the order $(111)<(110)<(100)$. Conceptually if one were to begin with a spherical crystal one could expect that etching would lead to a cubic structure as illustrated in Figure 5. The slower etch rate in (110) and (111) than that in (100) directions may generate the emergence of (100) surface on the crystal. Note
TABLE 1: Comparisons of MD Etching Rates with Experiments

\begin{tabular}{lcc}
\hline & experiments (from ref 16) & MD simulations \\
\hline$R(100) / R(111)$ & 1.47 & 1.53 \\
$R(100) / R(110)$ & 1.12 & 1.22
\end{tabular}

that in order to model the conditions under which the nonequilibrium plasma experiments were performed, it is interesting to observe morphology transition from a sphere to a cube by the etching. However, as stated in a previous section the simulation time is much higher due to the factor of 10 smaller time step to properly simulate hydrogen (light) atoms, and the existence of many local minima.

To address the issue of etching we prepare three Si surface samples $(100,110$, and 111) in an MD simulation cavity with periodic boundary conditions in $x$ and $y$ directions. The surface was composed of 8 atoms layers which were simulated at constant energy, and were situated on top of 10 layers of atoms thermostatted to a constant temperature. This structure was held by another 2 atom layers that held fixed. The top layer was equilibrated at $700 \mathrm{~K}$ with a saturated layer of hydrogen. A hydrogen atom is introduced at a random $x-y$ location every 2 ps and was fired at the substrate, starting from a distance of 1 $\mathrm{nm}$ above the surface, with the kinetic energy corresponding to $700 \mathrm{~K}$. By counting the number of vaporized Si atoms we can compute the relative etch rate for each of the three crystal faces. The resulting ratios of etch rate are shown in Table 1. These results are in excellent agreement with values obtained by Sasaki and Takada. ${ }^{53}$ This result is consistent with the idea that crystals formed by rapid vapor phase nucleation can undergo a rapid morphological transformation to the lowest energy crystal configuration thorough etching processes. In the particular case of silicon, the cubic structure comes about because of two constraints. One that the cubic structure is energetically favored when hydrogen terminated, and two that the plasma which operates with hydrogen also provides a steady source of hydrogen atoms for etching.

\section{Conclusion}

The energetics of various shaped Si nanocrystals and the role of hydrogen surface termination in nonequilibrium plasma are considered. From our MD simulations, the cubic structure comes about because 1) the cubic structure is energetically favored when hydrogen terminated and 2) the plasma that operates with high concentration of hydrogen also provides a steady source of hydrogen atoms for etching.

Acknowledgment. The authors thank Prof. Uwe Kortshagen for the very insightful and fruitful discussions. This work is supported by NSF Grant No. CTS-0083062, the Army High Performance Computing Research Center DAAD19-01-2-0014 and the Minnesota Super-Computer Center.

\section{References and Notes}

(1) Alivisatos, A. P. Science 1996, 271, 933.

(2) Colvin, V. L.; Alivisatos, A. P.; Tobin, J. G. Phys. Rev. Lett. 1991, $66,2786$.

(3) Goldstein, A. N.; Echer, C. M.; Alivisatos, A. P. Science 1992, 256,1425 .

(4) Shi, J.; Gider, S.; Babcock, K.; Awschalom, D. D. Science 1996, $271,937$.

(5) Fu, Y.; Willander, M.; Dutta, A.; Oda, S. Superlattices Microstruct. 2000, 28, 177 .

(6) Nishiguchi, K.; Oda, S. J. Appl. Phys. 2000, 88, 4186.

(7) Tiwari, S.; Rana, F.; Hanafi, H.; Hartstein, A.; Crabbe, E. F.; Chan, K. Appl. Phys. Lett. 1996, 68, 1377. 
(8) Tiwari, S.; Rana, F.; Chan, K.; Shi, L.; Hanafi, H. Appl. Phys. Lett. 1996, 69, 1232.

(9) Ostraat, M. L.; De Blauwe, J. W.; Green, M. L.; Bell, L. D.; Brongersma, M. L.; Casperson, J.; Flagan, R. C. Appl. Phys. Lett. 2001, $79,433$.

(10) Banerjee, S.; Huang, S.; Yamanaka, T.; Oda, S. J. Vac. Sci. Technol. $B$ 2002, 20, 1135 .

(11) Littau, K. A.; Szajowski, P. J.; Muller, A. J.; Kortan, A. R.; Brus,

L. E. J. Phys. Chem. 1993, 97, 1224.

(12) Collins, R. T.; Fauchet, P. M.; Tischler, M. A. Phys. Today 1997, $50,24$.

(13) Nayfeh, M.; Akcakir, O.; Therrien, J.; Yamani, Z.; Barry, N.; Yu, W.; Gratton, E. Appl. Phys. Lett. 1999, 75, 4112.

(14) St, J.; Coffer, J. L.; Chen, Y. J. Am. Chem. Soc. 1999, 121, 1888

(15) Canham, L. T. Nature 2000, 408, 411.

(16) Ledoux, G.; Guillois, O.; Porterat, D.; Reynaud, C.; Huisken, F.; Kohn, B.; Paillard, V. Phys. Rev. B 2000, 62, 15942.

(17) Borsella, E.; Falconieri, M.; Sangaletti, L.; Allieri, B.; Depero, L.

Mater. Sci. Eng. B 2001, 79, 55.

(18) Holmes, J. D.; Ziegler, K. J.; Doty, C.; Pell, L. E.; Johnston, K. P.; Korgel, B. A. J. Am. Chem. Soc. 2001, 123, 3743.

(19) Park, N. M.; Kim, T. S.; Park, S. J. Appl. Phys. Lett. 2001, 78, 2575 .

(20) Franzo, G.; Irrera, A.; Moreira, E. C.; Miritello, M.; Iacona, F.; Sanfilippo, D.; Di Stefano, G.; Fallica, P. G.; Priolo, F. Appl. Phys. A: Mater. Sci. Process. 2002, 74, 1.

(21) Ledoux, G.; Gong, J.; Huisken, F.; Guillois, O.; Reynaud, C. Appl. Phys. Lett. 2002, 80, 4834.

(22) Pettigrew, K. A.; Liu, Q.; Power, P. P.; Kauzlarich, S. M. Chem. Mater. 2003, 15, 4005.

(23) Canham, L. T. Appl. Phys. Lett. 1990, 57, 1046.

(24) Nayfeh, M.; Barry, N.; Therrien, J.; Akcakir, O.; Gratton, E.; Belomoin, G. Appl. Phys. Lett. 2001, 78, 1131.

(25) Baldwin, R. K.; Pettigrew, K. A.; Garno, J. C.; Power, P. P.; Liu,

G. Y.; Kauzlarich, S. M. J. Am. Chem. Soc. 2002, 124, 1150.

(26) Ostraat, M. L.; Blauwe, W. D.; Green, M. L.; Bell, L. D.; Atwater,

H. A.; Flagan, R. C. J. Electrochem. Soc. 2001, 148, G265.

(27) Bapat, A.; Anderson, C.; Perrey, C. R.; Carter, C. B.; Campbell,

S. A.; Kortshagen, U. Plasma Phys. Controlled Fusion 2004, 46, B97.
(28) Sayle, T. X. T.; Parker, S. C.; Sayle, D. C. Phys. Chem. Chem. Phys. 2005, 7, 2936.

(29) Freeman, D. L.; Doll, J. D. Annu. Rev. Phys. Chem. 1996, 47, 43.

(30) Johnston, R. L. Dalton Trans. 2003, 22, 4193.

(31) Zhao, Y.; Kim, Y. H.; Du, M. H.; Zhang, S. B. Phys. Rev. Lett. 2004, 93, 015502.

(32) Combe, N.; Jensen, P.; Pimpinelli, A. Phys. Rev. Lett. 2000, 85, 110.

(33) Barnard, A.; Zapol, P. J. Chem. Phys. 2004, 121, 4276.

(34) Cleveland, C. L.; Landman, U. J.; Schaaff, T. G.; Shafigullin, M. N.; Stephens, P. W.; Whetten, R. L. Phys. Rev. Lett. 1997, 79, 1873.

(35) Michaelian, K.; Rendon, R.; Garzon, I. L. Phys. Rev. B 1999, 60, 2000 .

(36) Cleveland, C. L.; Luedtke, W. D.; Landman, U. J. Phys. Rev. B 1999, 60, 5065.

(37) Wang, Y.; Teitel, S.; Dellago, C. J. Chem. Phys. 2005, 122, 214722.

(38) Hawa, T.; Zachariah, M. R. Phys. Rev. B 2004, 69, 035417.

(39) Stillinger, F. H.; Weber, T. S. Phys. Rev. B 1985, 31, 5262.

(40) Kohen, D.; Tully, J. C.; Stillinger, F. H. Surf. Sci. 1998, 397, 225.

(41) Murty, M. V. R.; Atwater, H. A. Phys. Rev. B 1995, 51, 4889.

(42) T. Ohira., T. Inamuro., and T. Adachi., in Mater. Res. Soc. Symp.

Proc., San Francisco, 1994, Vol. 336, p. 177.

(43) Ohira, T.; Ukai, O.; Adachi, T.; Takeuchi, Y.; Murata, M. Phys. Rev. B 1995, 52, 8283.

(44) Ohira, T.; Ukai, O.; Noda, M. Surf. Sci. 2000, 458, 216.

(45) Ramalingam, S.; Maroudas, D.; Aydil, E. S. J. Appl. Phys. 1998, 84,3895 .

(46) Tersoff, J. Phys. Rev. B 1988, 38, 9902.

(47) Tersoff, J. Phys. Rev. B 1986, 37, 6991.

(48) Tersoff, J. Phys. Rev. Lett. 1988, 56, 632.

(49) Tersoff, J. Phys. Rev. B 1989, 39, 5566.

(50) Verlet, L. Phys. Rev. B 1967, 159, 98. 655 .

(51) Mangolini, L.; Thimsen, E.; Kortshagen, U. Nano Lett. 2005, 5,

(52) Jurbergs, D.; Rogojina, E.; Mangolini, L.; Kortshagen, U. Appl. Phys. Lett. 2006, 88, 233116.

(53) Sasaki, K.; Takada, T. Jpn. J. Appl. Phys. 1998, 37, 402.

(54) Kortshagen, U.; Mangolini, L.; Bapat, A. J. Nanopart. Res. 2007, 9,39 .

\section{JP800780S}

